\title{
Paraclival or Cavernous Internal Carotid Artery: One Segment but Two Names
}

\author{
Puya Alikhani ${ }^{1}$ Sananthan Sivakanthan ${ }^{1}$ Harry van Loveren ${ }^{1}$ Siviero Agazzi ${ }^{1}$
} ${ }^{1}$ Department of Neurosurgery and Brain Repair, University of South
Florida, Florida, United States

J Neurol Surg B 2016;77:304-307.
Address for correspondence Sananthan Sivakanthan, BS, Department of Neurosurgery and Brain Repair, 2 Tampa General Circle, Tampa, FL 33606, United States (e-mail: ssivakan@health.usf.edu).

\begin{abstract}
Keywords

- transclival ICA

- vidian nerve

- petrolingual ligament

- lacerum ICA

Background The anatomy of the carotid artery has traditionally been understood into segments that correspond to the surrounding relevant anatomy. Transcranial surgery favors the seven segments classification initially posited by our group in 1996. With the advent of endoscopic approaches a new term has been added to the carotid anatomy lexicon "the paraclival internal carotid artery (ICA)." This "paraclival" carotid is a very familiar segment for all endoscopically trained skull base surgeons but a clear correlate to the transcranial anatomy has not been described. The purpose of our study is to improve the communication between endoscopic and open trained skull base surgeons by correlating the endoscopic paraclival ICA to the transcranial segments of the ICA. Methods Two cadaveric specimens underwent both endoscopic and transcranial dissection. Aneurysm clips were placed in the proximal and the distal extent of the paraclival ICA as described in previous literature. The clip positions were visualized and correlated to open anatomical landmarks.

Results The proximal clip was located just medial to the origin of the petrolingual ligament, while the distal clip was placed just inferior to the posterior genu of the cavernous ICA.

Conclusion The paraclival ICA corresponds to lacerum segment of ICA and vertical cavernous ICA.
\end{abstract}

\section{Introduction}

The advent of endoscopic skull base surgery has forever changed the neurosurgeon's perspective of the skull base. Transcranial approaches, "from above," and endoscopic approaches, "from below," offer different views of the same anatomy. During this paradigm shift some anatomical concepts have been reconstructed to better fit the endoscopic mind frame. The internal carotid artery (ICA) serves as an important landmark in both open and endoscopic skull base surgery. Thus, the nomenclature of the different segments of the ICA is a crucial point of understanding for skull base surgeons.

In this article, we study the segment of ICA referred to as "paraclival" in the expanded endoscopic literature and correlate its anatomy with the traditional neurosurgical classification described by Bouthillier et al ${ }^{1}$ in 1996.

\section{Materials and Methods}

Institutional review board approval was obtained for use of cadaveric specimens for dissection. Two fresh cadaveric heads were injected with colored liquid latex. ${ }^{2}$ An expanded endoscopic approach to the clivus was performed with $4 \mathrm{~mm}, 18 \mathrm{~cm}$, and 0,30 , and 45-degree endoscope, scopes, coupled with high-definition

(c) 2016 Georg Thieme Verlag KG Stuttgart · New York
DOI http://dx.doi.org/ 10.1055/s-0035-1568870. ISSN 2193-6331. 
camera and monitor (Karl Storz Endoscopy, Tuttlingen, Germany).

\section{Dissection Technique}

A middle turbinectomy was performed on one side to assist with binostril work. The sphenoid ostium above the sphenoethmoidal recess was identified and a posterior septectomy was performed. The sphenoid rostrum was removed in its entirety. The sellar floor, medial opticocarotid recesses, and lateral opticocarotid recesses were identified and drilled. The sellar dura was opened and the pituitary gland was identified in the midline. Cavernous sinus was opened and abducent nerve was identified. Then the ICA was dissected distally from its supraclinoid segment inferiorly toward the foramen lacerum (FL) proximally.

Attention was then brought lateral the middle turbinate to expose the pterygopalatine fossa (PPF) The maxillary ostium was identified and opened medial to the middle turbinate. The posterior wall of maxillary sinus (PWMS) was resected to gain access to PPF. The fat pad posterior to PWMS was removed and the branches of the third division of the internal maxillary artery were identified and lateralized. The vidian nerve and maxillary nerve (V2) were identified anteromedially and posterolaterally, respectively. The vidian nerve was followed posteriorly toward the petrous apex where it reaches the

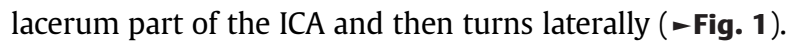

Two aneurysm clips were then used to mark the paraclival ICA (-Fig. 2). One clip was placed immediately above the fibrocartilage marking the anterior genu of the petrous ICA (also known as lacerum segment of the ICA) and the other one just below the abducent nerve.

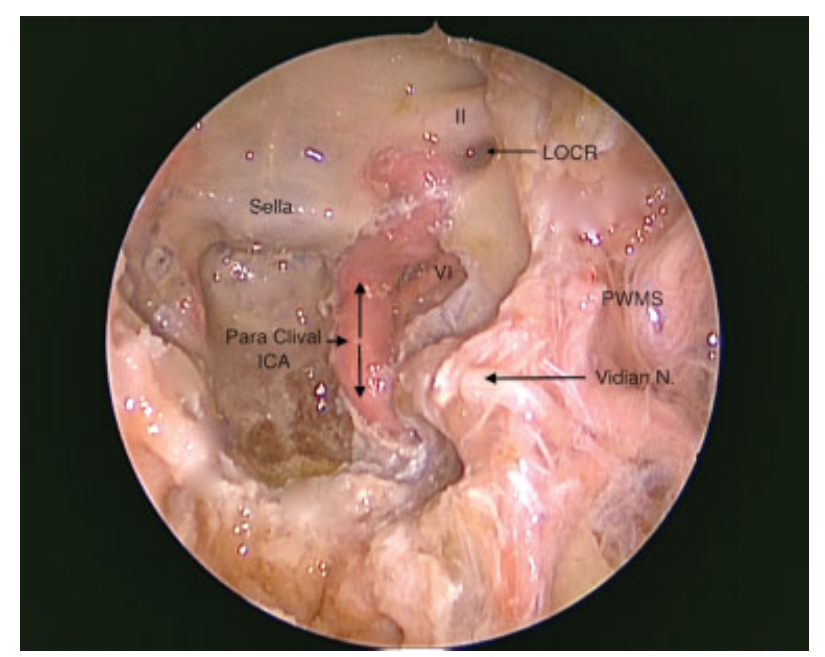

Fig. 1 Endoscopic dissection of left paraclival ICA: This view was obtained after a bilateral sphenoidotomy, removal of the rostrum, left maxillary antrostomy, and removal of the posterior wall of maxillary sinus. In this dissection one can see the optic nerve, LOCR, which the pneumatized optic strut, sellar ICA, Paraclival ICA, abducens nerve, and the vidian nerve. The vidian nerve can be localized in the PWMS at the junction of medial pterygoid plate and PPF. Vidian nerve runs in the floor of sphenoid sinus and can be track back to lacerum ICA where it turns laterally to stay superior to petrous ICA. ICA, internal carotid artery; LOCR, lateral opticocarotid recess; PPF, pterygopalatine fossa; PWMS, posterior wall of maxillary sinus.

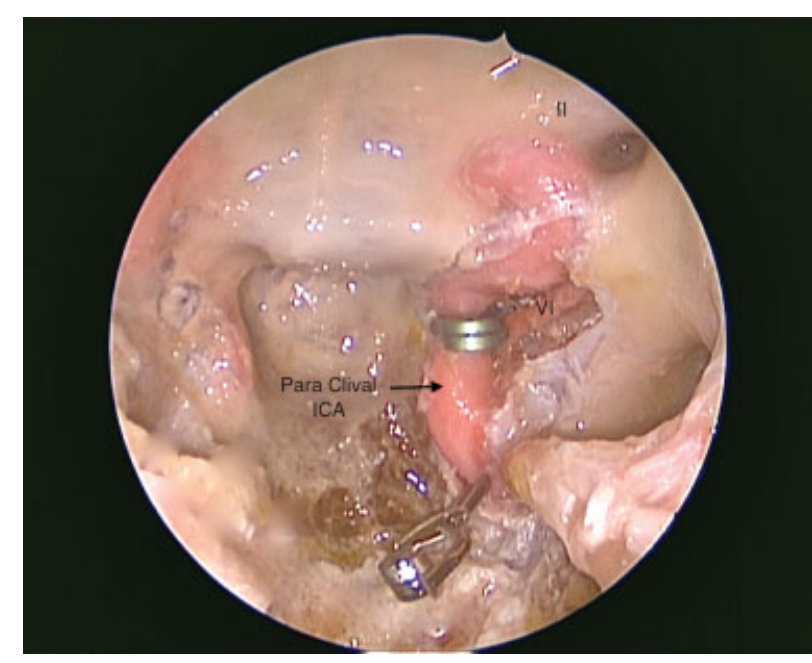

Fig. 2 Endoscopic dissection of left paraclival ICA: This view was obtained after a bilateral sphenoidotomy, removal of the rostrum, left maxillary antrostomy and removal of the posterior wall of maxillary sinus. Two aneurysm clips were placed. Proximal clip (direction of the blood flow) was placed at the lacerum segment of ICA. It is worth emphasizing the tips of the clips were not encircling the entire ICA thickness due to the presence of PLL posteriorly. This clip was advance later when PLL was cut and removed transcranially. Distal clip was placed just above the abducens nerve (CN VI). ICA, internal carotid artery, PLL, petrolingual ligament.

Each head was then opened transcranially with a standard pterional craniotomy. The anterior and middle fossa dura was removed to facilitate exposure of the cavernous sinus and the Gasserian ganglion ( - Fig. 3). The root of the trigeminal nerve was cut at the porus trigeminus and the Gasserian ganglion was reflected anteriorly to expose the petrolingual ligament $(\mathrm{PLL})$ and the petrosphenoidal ligament (PSL) ( - Fig. 4). Then the PLL was cut and removed. At this time, we were able to

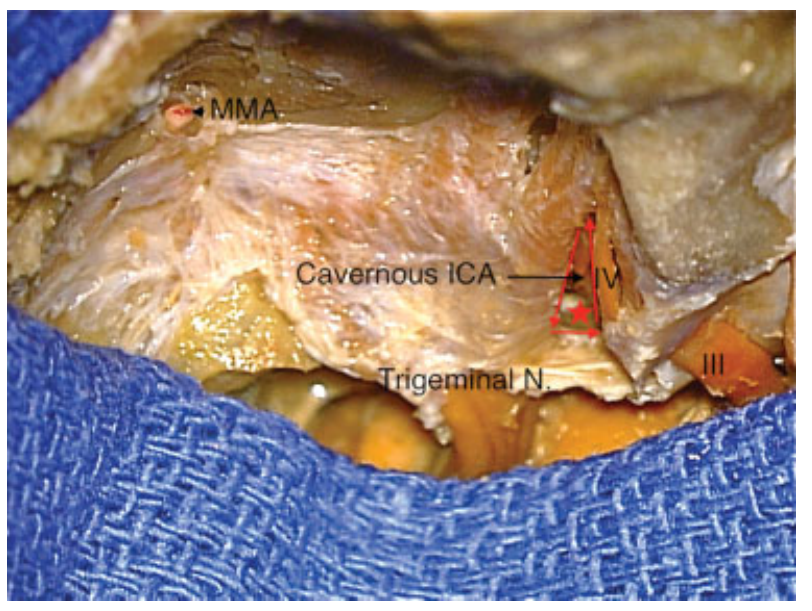

Fig. 3 Transcranial exposure of ICA: This view was obtained after standard pterional craniotomy. In this dissection one can see the intradural ICA, oculomotor nerve (CN III), trochlear nerve (CN IV), trigeminal nerve (CN V), and MMA. Left cavernous sinus was dissected. Parkinson triangle (infratrochlear triangle) was identified (red star). Vertical cavernous ICA and abducens nerve (CN VI) was visualized inside this triangle. ICA, internal carotid artery; MMA, middle meningeal artery. 


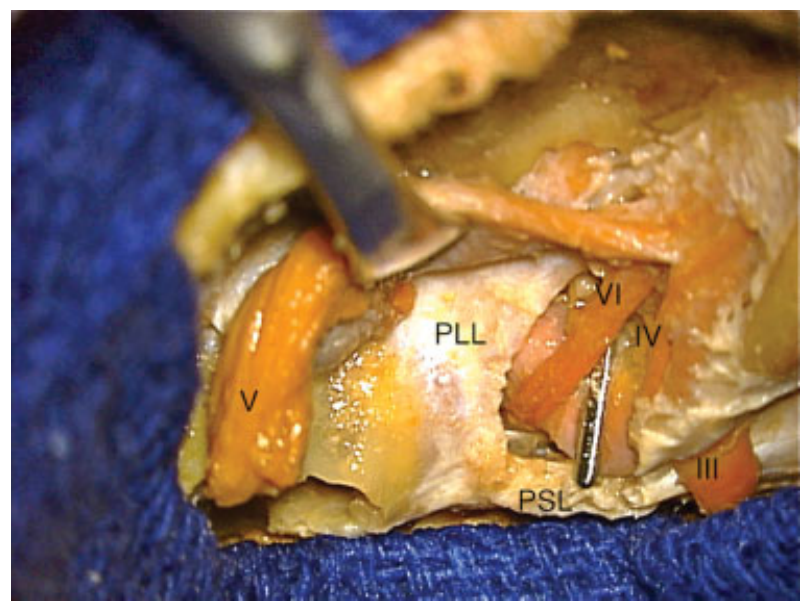

Fig. 4 Transcranial exposure of ICA: This view was obtained after standard pterional craniotomy. In this dissection one can see the intradural ICA, oculomotor nerve (CN III), trochlear nerve (CN IV), trigeminal nerve (CN V), and MMA. Trigeminal nerve was cut at its cisternal segment and was lifted anteriorly. Petrosphenoid ligament (PSL, Gruber ligament) and PLL was identified. ICA, internal carotid artery; MMA, middle meningeal artery; PSL, petrosphenoid ligament; PLL, petrolingual ligament.

visualize, from a transcranial approach, both clips placed endoscopically ( - Fig. 5). Their exact location on the carotid artery was recorded and photographed (- Fig. 6).

\section{Results}

Our dissection confirmed that from a transcranial approach, the proximal (inferior) clip was located just medial to the origin of the petrolingual ligament, while the distal clip was placed just inferior to the posterior genu of the cavernous ICA ( - Fig. 6 ). The two clips were therefore defining the lacerum segment and the vertical portion of the cavernous segment ICA.

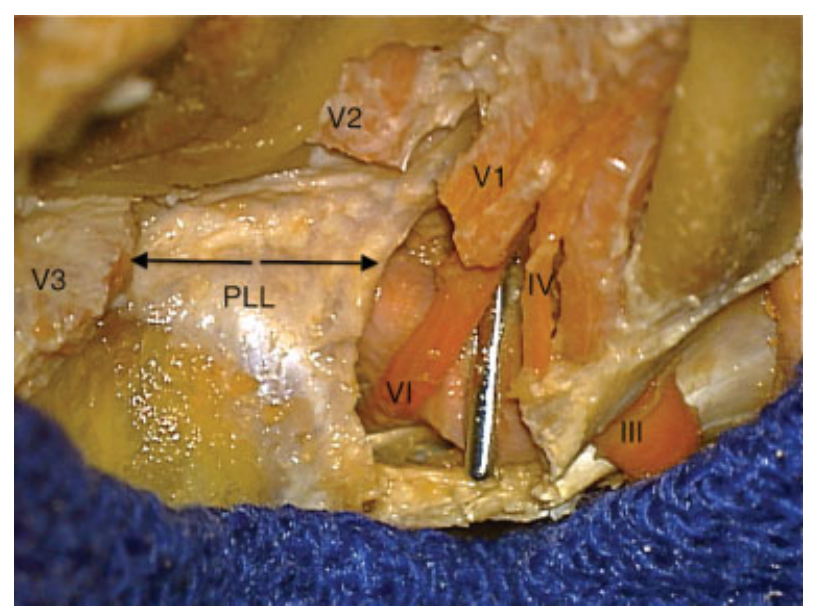

Fig. 5 Transcranial exposure of ICA: This view was obtained after standard pterional craniotomy. In this dissection one can see the intradural ICA, Oculomotor nerve (CN III), trochlear nerve (CN IV), trigeminal nerve $(C N V)$, and . Trigeminal nerve was cut and removed and entire PLL was exposed. ICA, internal carotid artery; MMA, middle meningeal artery; PLL, petrolingual ligament.

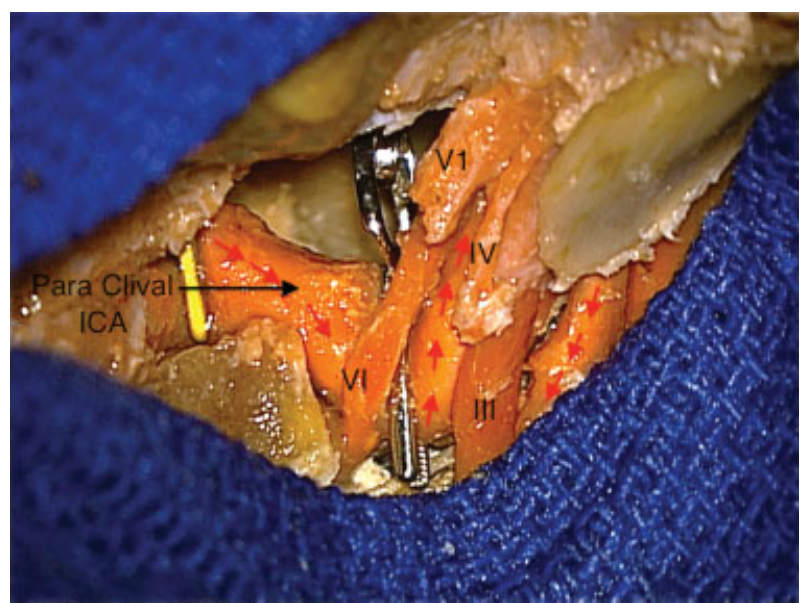

Fig. 6 Transcranial exposure of ICA: This view was obtained after standard pterional craniotomy. In this dissection one can see the intradural ICA, oculomotor nerve (CN III), trochlear nerve (CN IV), trigeminal Nerve (CN V), and MMA. To expose the distal clip, PLL was cut and dissected out. Paraclival ICA that is the lacerum ICA and vertical segment of cavernous ICA can be seen. ICA, internal carotid artery; MMA, middle meningeal artery; PLL, petrolingual ligament.

\section{Discussion}

Our dissection demonstrates that the segment of the ICA referred to as "paraclival" in the expanded endonasal literature corresponds to the lacerum and vertical part of the cavernous segment of the ICA as defined by Bouthillier et al in 1996. The concept of a paraclival ICA is a relatively new term in the carotid classification and this is the first anatomical study to correlate the endoscopic and transcranial nomenclature.

Like transcranial surgery, endoscopic approaches to the skull base have put a significant emphasis on ICA anatomy and for this reason, it is important to clarify the endoscopic and the transcranial nomenclature to allow for accurate communication in the operating room.

There have been several studies and anatomical dissections developing an understanding of the ICA. In 1938, Fischer ${ }^{3}$ described segments of the ICA, $\mathrm{C} 1$ to $\mathrm{C}$, based on the angiographic course of ICA. Transcranial surgery favors the seven segments classification initially posited by our group. In this description the ICA segments were named according to their surrounding anatomy and the compartments through which they travel. The ICA was divided into seven segments: $\mathrm{C}$, cervical; C2, petrous; C3, lacerum; C4, cavernous; C5, clinoid; $\mathrm{C} 6$, ophthalmic; and $\mathrm{C}$, communicating. This classification has been widely used in the neurosurgical literature.

The definition of the lacerum ICA, which runs posterior to the petrolingual ligament remains controversial in neurosurgery. Bouthillier et al defined this segment from the end of the carotid canal to the anterior edge of PLL. Ziyal et al, ${ }^{4}$ on the other hand, called this segment a trigeminal segment of ICA because it was hidden below the Gasserian ganglion. This group did not accept the term lacerum ICA because the artery never travels inside FL. Although both groups had their point of view, the anatomy of the FL and PLL remains crucial for 
understanding this segment of the ICA. FL is formed by the confluence of the basilar occiput, petrous apex, and sphenoid bone. It measures $5.7 \mathrm{~mm}$ (range, 4.3-9.1 $\mathrm{mm}$ ) in anteroposterior diameter and $5.7 \mathrm{~mm}$ (range, $4.3-9.1 \mathrm{~mm}$ ) in medial to lateral diameter. ${ }^{5}$ The PLL spans from the lingual process of the sphenoid bone to the petrous apex transcranially and covers the ICA over FL. In expanded endoscopic approaches, the PLL is hidden posterior to the ICA. Endoscopically this segment of ICA is visible at the end of the carotid canal.

The definition of the intracavernous portion of the ICA seems to be more consistent in the literature. It is divided into a posterior vertical, posterior bend, anterior bend, and anterior vertical segments. ${ }^{6,7}$ This segment starts from the anterior edge of the PLL and ends at the proximal dural ring.

Endoscopically, the paraclival ICA lies along the clivus. It starts at the FL, where it runs anterior to the PLL and terminates at the posterior bend of the cavernous ICA, just above the entry of the sixth cranial nerve into the cavernous sinus. ${ }^{8}$ Alfieri and $\mathrm{Jho}^{9}$ in 2001 , divided the paraclival ICA in two parts, the lacerum segment caudally and the trigeminal segment rostrally. This was due to the fact that the paraclival ICA runs alongside the trigeminal nerve in Meckel cave endoscopically. Other groups have called the paraclival ICA the vertical segment of cavernous ICA. ${ }^{10-12}$

This report demonstrates a key anatomical nuance using a simple and direct methodology.

\section{Conclusion}

The ICA plays a very important anatomical landmark in both endoscopic endonasal surgery and open transcranial procedures. Detailed anatomical knowledge of the ICA can guide the surgeon in endoscopic approaches to the skull base. In this article, we present a transcranial correlation to the endoscopic definition of the paraclival ICA.

\section{References}

1 Bouthillier A, van Loveren HR, Keller JT. Segments of the internal carotid artery: a new classification. Neurosurgery 1996;38(3): 425-432, discussion 432-433

2 Alvernia JE, Pradilla G, Mertens P, Lanzino G, Tamargo RJ. Latex injection of cadaver heads: technical note. Neurosurgery 2010;67 (2, Suppl Operative):362-367

3 Fischer E. Die Lageabweichungen der vorderen hirnarterie im gefässbild. Zentralbl Neurochir 1938;3:300-313

4 Ziyal IM, Ozgen T, Sekhar LN, Ozcan OE, Cekirge S. Proposed classification of segments of the internal carotid artery: anatomical study with angiographical interpretation. Neurol Med Chir (Tokyo) 2005;45(4):184-190, discussion 190-191

5 Osawa S, Rhoton AL Jr, Seker A, Shimizu S, Fujii K, Kassam AB. Microsurgical and endoscopic anatomy of the vidian canal. Neurosurgery 2009;64(5, Suppl 2):385-411, discussion 411412

6 Jittapiromsak P, Sabuncuoglu H, Deshmukh P, McDougall CG, Spetzler RF, Preul MC. Anatomical relationships of intracavernous internal carotid artery to intracavernous neural structures. Skull Base 2010;20(5):327-336

7 Tubbs RS, Hansasuta A, Loukas M, et al. Branches of the petrous and cavernous segments of the internal carotid artery. Clin Anat 2007; 20(6):596-601

8 Fortes FSG, Pinheiro-Neto CD, Carrau RL, Brito RV, Prevedello $\mathrm{DM}$, Sennes LU. Endonasal endoscopic exposure of the internal carotid artery: an anatomical study. Laryngoscope 2012;122(2): 445-451

9 Alfieri A, Jho HD. Endoscopic endonasal cavernous sinus surgery: an anatomic study. Neurosurgery 2001;48(4):827-836, discussion 836-837

10 Abuzayed B, Tanriover N, Gazioglu N, Akar Z. Extended endoscopic endonasal approach to the clival region. J Craniofac Surg 2010; 21(1):245-251

11 Cavallo LM, Cappabianca P, Messina A, et al. The extended endoscopic endonasal approach to the clivus and cranio-vertebral junction: anatomical study. Childs Nerv Syst 2007;23(6): 665-671

12 Solari D, Villa A, De Angelis M, Esposito F, Cavallo LM, Cappabianca P. Anatomy and surgery of the endoscopic endonasal approach to the skull base. Transl Med UniSa 2012;2:36-46 